\title{
Generation of a monoclonal antibody reactive to prefusion myocytes
}

\author{
Tomohiro Kurisaki $\cdot$ Aki Masuda $\cdot$ Shiho Nakagiri $\cdot$ \\ Yoshihiro Hayata $\cdot$ Motoki Kuhara • \\ Yoshiro Kishi · Atsuko Sehara-Fujisawa
}

Received: 7 March 2011/ Accepted: 26 April 2011/Published online: 20 May 2011

(C) The Author(s) 2011. This article is published with open access at Springerlink.com

\begin{abstract}
We established a novel monoclonal antibody, Yaksa that is specific to a subpopulation of myogenic cells. The Yaksa antigen is not expressed on the surface of growing myoblasts but only on a subpopulation of myogenin-positive myocytes. When Yaksa antigen-positive mononucleated cells were freshly prepared from a murine myogenic cell by a cell sorter, they fused with each other and formed multinucleated myotubes shortly after replating while Yaksa antigen-negative cells scarcely generated myotubes. These results suggest that Yaksa could segregate fusion-competent, mononucleated cells from fusionincompetent cells during muscle differentiation. The Yaksa antigen was also expressed in developing muscle and regenerating muscle in vivo and it was localized at sites of cell-cell contact between mono-nucleated muscle cells and between mono-nucleated muscle cells and myotubes. Thus, Yaksa that marks prefusion myocytes before myotube formation can be a useful tool to elucidate the cellular and molecular mechanisms of myogenic cell fusion.
\end{abstract}

Keywords Cell fusion - Myogenesis - Skeletal muscle . Prefusion

Electronic supplementary material The online version of this article (doi:10.1007/s10974-011-9247-8) contains supplementary material, which is available to authorized users.

T. Kurisaki $(\bowtie) \cdot$ A. Masuda $\cdot$ S. Nakagiri .

A. Sehara-Fujisawa

Field of Growth Regulation, Institute for Frontier Medical

Sciences, Kyoto University, 53 Shogoin-Kawahara-cho,

Sakyo-ku, 606-8507 Kyoto, Japan

e-mail: kurisaki@frontier.kyoto-u.ac.jp

Y. Hayata $\cdot$ M. Kuhara $\cdot$ Y. Kishi

MBL, 396-0002 Ina, Japan

\section{Introduction}

Myoblast fusion is essential to form the multi-nucleated muscle fibers that provide the contractile strength of skeletal muscle (Buckingham 2001; Buckingham 2006; Horsley and Pavlath 2004). It is also responsible for the postnatal maintenance, growth, repair, and regeneration of skeletal muscles (Cerletti et al. 2008; Rudnicki et al. 2008). Myoblast fusion follows an ordered set of events: recognition, adhesion, and plasma membrane union, which results in syncytium formation (Jansen and Pavlath 2008). During the last decade, intensive study using model animals including Drosophila, Zebrafish, chick, and mouse have yielded many insights into the fusion mechanism of myoblasts (Haralalka and Abmayr 2010; Moore et al. 2007; Rochlin et al. 2010; Mermelstein et al. 2005). It is also suggested that several of the signaling cascades involved in myoblast fusion are evolutionarily conserved (Buckingham 2001; Krauss 2010). However, relatively little is yet known about the molecular mechanism of membrane union.

Myogenic cell lines have been useful model systems for the study of muscle differentiation and cellular fusion. The murine myoblast $\mathrm{C} 2$ is a well-characterized myogenic cell line (Yaffe and Saxel 1977). Serum deprivation triggers a series of events that result in myogenic differentiation in $\mathrm{C} 2$ cells. Expression of myogenin (mg), a transcription factor critical for myotube formation, is the earliest event (Andres and Walsh 1996). After that, the cells withdraw from the cell cycle, followed by cellular fusion resulting in the formation of multinucleated myotubes (Halevy et al. 1995; Miller 1990; Parker et al. 1995). Only a fraction of C2 myoblasts undergo terminal differentiation upon serum deprivation, while others, known as reserve cells, do not follow the cellular events described above (Morgan and Partridge 2003; Yoshida et al. 1998). 
Myogenic cell fusion occurs asynchronously over a relatively long period of several days. This means that only a small population of fusion-competent cells exists at any specific point in time during differentiation, making elucidation of the cellular and molecular mechanisms of myogenic cell fusion difficult. Antibody $(\mathrm{Ab})$ that recognize fusion-competent cells alive before myotube formation, is one of the powerful tools, but not available so far. Here we report successful isolation of fusion-competent myocytes with a novel monoclonal antibody, Yaksa.

\section{Materials and methods}

Materials

Chemicals were purchased from Sigma (St. Louis, MO, USA) and Nacalai Tesque (Kyoto, Japan). Reagents for molecular biology were purchased from TaKaRa (Kyoto, Japan) and Toyobo (Osaka, Japan) unless otherwise indicated. DAPI, Hoechst 33342, Fluorochrome-conjugated Streptavidin (StAv) were purchased from Molecular Probes (Eugene, OR, USA).

\section{Antibodies}

The antibodies used and their sources were as follows. ALEXA488-conjugated goat anti-rat IgG which have been adsorbed against mouse IgG and mouse serum, was from Molecular Probes. Isotype-matched control IgG2a and IgG2a-bio, were from BD Pharmingen (San Diego, CA, USA). Anti-myogenin mAb (F5D) was from Santa Cruz (Santa Cruz, CA, USA). Anti-alpha actinin mAb (EA-53) was from Sigma. Anti-desmin (D9) was from Progen (Heidelberg, Germany). Cy3-conjugated goat anti-mouse IgG was from Amersham (Bucks, UK). Biotinylation of immunoglobulin was performed by using EZ-Link Sulfo NHS-LC-Biotin (Pierce, Rockford, IL, USA) according to the manufacturer's instructions.

\section{Animals}

Mice (C57BL6/J) and Wistar rats were purchased from Shimizu Laboratory Supplies (Kyoto, Japan) and maintained under specific pathogen-free conditions in environmentally controlled clean rooms at the Institute for Frontier Medical Sciences, Kyoto University. The experiments were conducted according to institutional ethical guidelines for animal experimentation and safety guidelines for gene manipulation experiments.
Cell culture

A subclone of C2 myoblasts, designated C2/4 (Yoshida et al. 1996), was used and is referred to simply as C2 in this study. The cells were routinely propagated in the growth medium, DMEM supplemented with $10 \%$ fetal calf serum (FCS), on collagen type I-coated culture plates (Iwaki, Japan). To induce differentiation, cells were seeded at a density of $2.5 \times 10^{4}$ cells/well (12-well plates) or the equivalent in growth medium, and $24 \mathrm{~h}$ later switched to the differentiation medium, DMEM supplemented with $2 \%$ horse serum (HS). Myotube formation was observed $72 \mathrm{~h}$ after the medium change. Primary myoblasts (pMB) were prepared from the gastrocnemius muscle of an 8-week-old mouse as described before (Hashimoto et al. 2004; Wada et al. 2002).

Stable transformant

mgEGFP-C2, the stable transformant used in this study, was generated using a pMG-dEGFP vector. The pMGdEGFP vector was generated by replacing the CMV promoter region of pd4EGFP-N1 (Clontech, Mountain View, CA, USA) with the upstream region of the myogenin gene (Fujisawa-Sehara et al. 1993). Transfections were performed using Lipofectamine PLUS (Invitrogen) according to the manufacturer's instructions. C2 cells were first transfected with pMG-dEGFP and selected by Geneticin (400 $\mu \mathrm{g} / \mathrm{ml}$, Invitrogen) to isolate several clones.

\section{Establishment of monoclonal antibodies}

A four-week-old female Wistar rat was immunized with GFP-expressing mgEGFP-C2 by using a differential footpad immunization protocol (Dzionek et al. 2000). Monoclonal antibodies (Abs) were established according to a standard procedure (Harlow and Lane 1988). The strategies for monoclonal antibody (mAb) selection are described in the "Results" section. Isotyping was performed using a Rat Monoclonal Antibody Isotyping Test Kit (Serotec, Oxford, UK) according to the manufacturer's instructions.

Flow cytometry and cell sorting

For flow cytometry, cells were dispersed with Accutase (Innovative Cell Technology, San Diego, CA, USA), stained and then analyzed using a FACScan analyzer and CellQuest software (BD, Mountain View, CA, USA). Dead cells were excluded from the plots based on propidium iodide staining. mgEGFP-C2 cells for immunization and Yaksa antibody-reactive cells were fractionated on a FACS Vantage or FACSAria cell sorter (BD). 
Replating assay

Thirty-six hours after induction of differentiation, mAbreactive cells were positively selected from $\mathrm{C} 2$ cells with biotinylated Yaksa mAb using either a FACS Vantage or IMagnet cell sorter. Yaksa antigen-negative cells were collected from the negatively selected fraction. Sorted cells were plated at $2 \times 10^{5}$ cells/well in 24 -well plates, cultured for 6-18 h, and examined by microscopy.

\section{Regeneration of muscle}

To induce muscle regeneration, 50-100 $\mu$ of cardiotoxin (CTX; $10 \mu \mathrm{M}$ in saline, Wako Chemicals) was injected into the muscle of 8-week-old mice. Regenerating musclederived mononucleated cells were prepared from C57BL/6 mice as previously described (Ojima et al. 2004).

\section{Immunostaining}

For immunostaining, PFA-fixed and permeabilized cells or unfixed cells were stained. Unfixed or PFA-fixed mouse embryos were cryosectioned, dried, and stained with hybridoma supernatant or antibodies. Tibialis anterior (TA) muscle was frozen in isopentane cooled with liquid nitrogen.

\section{Results}

Imaging expression of myogenin in live $\mathrm{C} 2$ cells

To generate monoclonal antibodies $(\mathrm{mAb})$ reactive to prefusion myocytes, we used $\mathrm{C} 2$ cells expressing myogenin as the immunogen. Myogenic cells acquire fusion competence only after the expression of myogenin, a muscle specific transcription factor that is essential for the development of skeletal muscle (Nabeshima et al. 1993). Only a fraction of $\mathrm{C} 2$ myoblasts express myogenin and undergo terminal differentiation after induction. Thus, enrichment of myogenin-expressing cells before fusion should enable efficient generation of monoclonal antibodies against prefusion myocytes. Then, we generated the cell line to visualize myogenin-expressing cells in living C2 cells. We previously showed that the region spanning $4 \mathrm{~kb}$ upstream of the myogenin gene is sufficient to mimic expression of myogenin mRNA during development (Fujisawa-Sehara et al. 1993). C2 cells were transiently transfected with pMG-dEGFP, a plasmid in which expression of destabilized GFP was driven by the $4 \mathrm{~kb}$ regulatory region of the myogenin gene, and stable transformants were established according to the following criteria. First, GFP is undetectable when cultured in growth medium. Second, GFP is activated after induction of differentiation. Third, the expression profile of GFP correlates with that of endogenous myogenin. mgEGFP-C2, was one of the stable transformants that satisfied those criteria. mgEGFP-C2 formed myotubes after induction of differentiation similar to the original $\mathrm{C} 2$ cells (data not shown).

As shown in Fig. 1, immunofluorescent staining reveals that neither GFP nor myogenin is expressed in growing mgEGFP-C2 and that GFP-positive cells also express myogenin at $48 \mathrm{~h}$ after myogenic induction. As determined by immunofluorescent staining, 10-20\% of mgEGFP-C2 cells express myogenin at $12-48 \mathrm{~h}$ after induction, and more than $85 \%$ of myogenin-positive cells express GFP at these time points. GFP-positive/myogenin-negative cells were hardly detectable (Fig. 1, data not shown). Therefore, we assumed that the expression of EGFP correlated with that of myogenin in mgEGFP-C2 cells at 12-48 $\mathrm{h}$ after induction. Then we generated rat monoclonal antibodies by
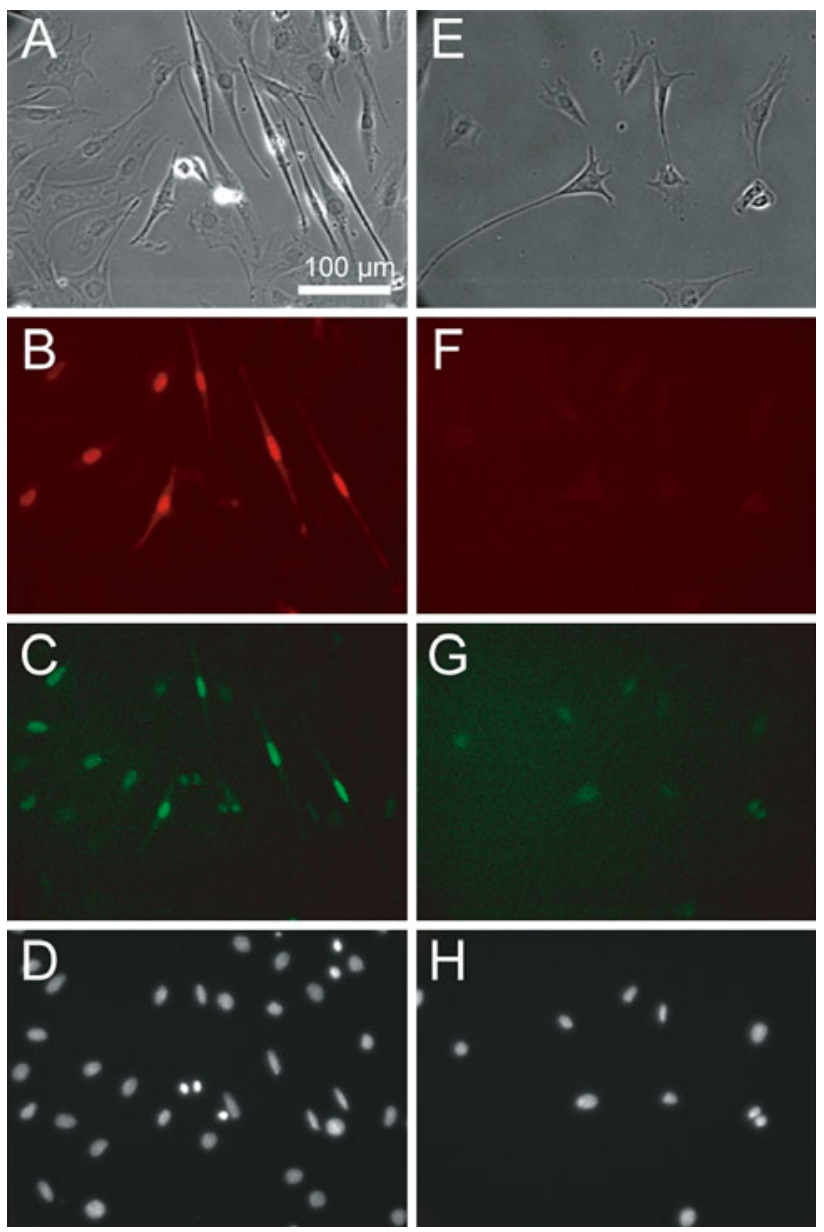

Fig. 1 High correlation between GFP and myogenin expression in mgEGFP-C2. mgEGFP-C2 culture at 2 days after induction of differentiation (A-D), and in 10\%CS-DMEM $(\mathbf{E}-\mathbf{H})$ was stained. $\mathbf{A}, \mathbf{E}$ : phase contrast; $\mathbf{B}, \mathbf{F}$ : anti-myogenin staining; $\mathbf{C}, \mathbf{G}$ : GFP; $\mathbf{D}, \mathbf{H}$ : DAPI staining 
differential footpad immunization with mgEGFP-C2 as described in "Materials and Methods".

Screening of monoclonal antibodies that detect fusion-competent myocytes

We screened almost 300 mAbs under the following criteria (Fig. 2). First, mAbs against prefusion myocytes react with a part of $\mathrm{C} 2$ cells after induction because only a subpopulation of $\mathrm{C} 2$ cells commit themselves to fusion-competent cells. Second, mAbs against prefusion myocytes enable efficient isolation of prefusion myocytes. If most of the cells sorted out with a mAb form myotubes, we assumed that the $\mathrm{mAb}$ reacted with prefusion myocytes. Last, the mAbs also react with embryonic muscle on the assumption that embryonic muscle and muscle satellite cells essentially share the same molecular machinery for myotube formation. Finally, we established eight mAbs that satisfied the above criteria. Yaksa was one of them. Yaksa refer to the nature-spirit among eight guardians for Buddhism. The isotype of Yaksa was IgG2a. The flow cytometric profile showed that Yaksa did not react with undifferentiated C2 cells and did react with only $20-30 \%$ of differentiating C2 cells (Fig. 3A). To characterize Yaksa-positive cells, $\mathrm{C} 2$ cells after myogenic induction for 1.5 days ( $36 \mathrm{~h})$, were double-stained with anti-myogenin $\mathrm{Ab}$ and Yaksa and analyzed using a FACScan. As shown in Fig. 3B, a subpopulation of myogenin-positive myocytes were Yaksapositive cells. When we performed a replating assay as described in "Materials and methods", most of the Yaksapositive cells formed myotubes 6-8 h after replating (Fig. 3C, S1) while Yaksa antigen (Ag)-negative cells and unsorted cells had formed only a few myotubes at the same time point. This indicates that Yaksa either recognize or isolate a subpopulation of cells that fuse with one another synchronously out of heterogeneously differentiating cell culture. Neither Yaksa Ag-negative cells nor unsorted cells formed more than a few myotubes even $36 \mathrm{~h}$ after replating. This suggests that Yaksa recognize fusion competency, not developmental delay, in this replating assay, and we concluded that Yaksa is a monoclonal $\mathrm{Ab}$ reactive to prefusion myocytes.

Yaksa antigen expression in vivo

Next, we investigated the expression profile of Yaksa Ag in vivo. Yaksa Ag was expressed in trunk at embryonic day (E) 13.5 (Fig. 4A). Yaksa stained tissue was also counterstained with anti-desmin Ab (Fig. 4B) and anti-myosin heavy chain $\mathrm{Ab}$ (data not shown). Then we concluded that Yaksa Ag was expressed in developing muscle. Yaksa Ag was also expressed in regenerating muscles (Fig. 4D-F). The tibialis anterior (TA) muscles were experimentally

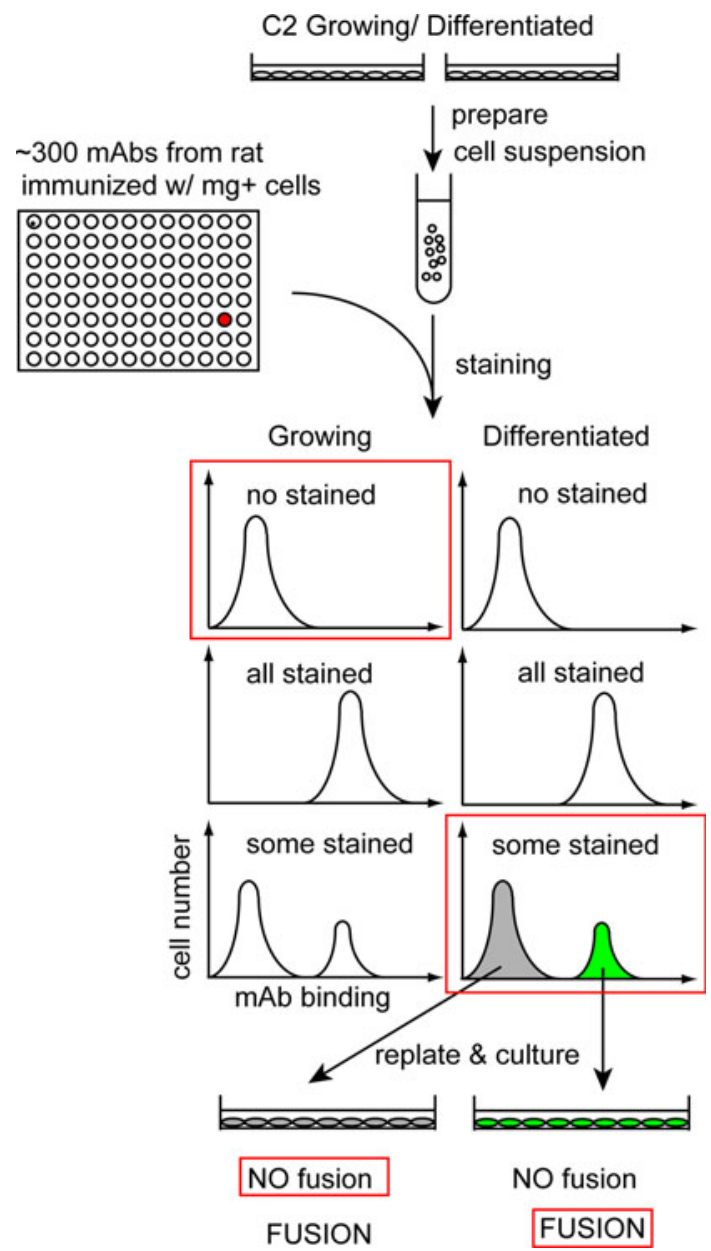

Fig. 2 Screening procedure. Mononucleated cell suspension was prepared from either growing or differentiated $\mathrm{C} 2$, stained with mAbs generated from rat immunized with myogenin-positive cells, and sorted. Staining pattern was categorized to three groups: No stained, All stained, and Some stained. We selected a mAb which does not stain growing $\mathrm{C} 2$ but some $\mathrm{C} 2$ cells after differentiation (red boxed). Then if the cells reactive with a mAb form myotubes efficiently by a replating assay (red boxed), we assumed that the mAb reacted with prefusion myocytes. For details, see "Materials and methods", and "Results"

damaged by cardiotoxin (CTX) injection to induce muscle regeneration (Hirata et al. 2003). The number of mononucleated cells in injured areas increased significantly following CTX injection, with a peak around day 3. The increase in cell number around day 3 is mainly attributable to proliferation of myogenic cells. Regenerating myotubes with central nuclei started to appear at day 3 and became more evident at days 5-7 post-injection. As shown in Fig. 4D-F and Fig. S2, Yaksa Ag was expressed in the plasma membrane of developing myotubes at days 3-5 after CTX injection. We did not detect Yaksa at days 0-2 and days 6-7 (Fig. S2, data not shown). These data suggest that Yaksa was expressed on fusing cells. Yaksa-positive cells were found in single-cell suspensions prepared from 
A
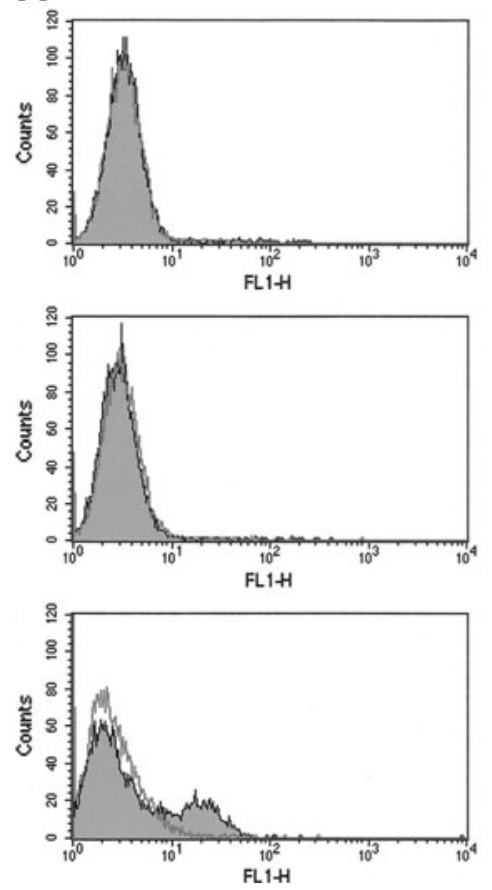

Fig. 3 Characterization of Yaksa. A FACScan analysis of Yaksa antigen on $\mathrm{C} 2$ cells. Upper growing, middle just before induction, lower 1.5 days after induction, dashed line control IgG2a. B Subpopulation of myogenin-positive cells express Yaksa antigen. FL1: myogenin stained with Alexa488, FL2; Yaksa antigen stained with PE. Thirty-six hours after induction, $\mathrm{C} 2$ cells were stained with control IgG (Upper Left), anti mg Ab (Lower Left), Yaksa (Upper

regenerating muscle at day 4 after CTX injection (Fig. 4G). We also confirmed Yaksa Ag expression in primary myoblasts prepared from adult mouse (Fig. $4 \mathrm{H}$ ). The culture apparently contained two cell types, that is Yaksa-positive/ high cells and Yaksa-negative/low cells. We presumed that the prepared primary myoblast culture contained prefusion myocytes already. As in the case of $\mathrm{C} 2$ cells, the amount of Yaksa $\mathrm{Ag}$ expression in individual cells correlated with their fusion competence. Primary myoblasts highly expressing Yaksa $\mathrm{Ag}$ fused with each other as early as $3 \mathrm{~h}$ after replating, much earlier than Yaksa-low myoblasts (data not shown). Yaksa did not react with several nonmyogenic cell lines including osteoclast-precursor cell lines, fibroblasts, hematopoietic cells, and ES cells (data not shown).

Yaksa localization on fusing myoblasts

To determine the localization of Yaksa Ag, pMB was transduced using a retrovirus vector carrying GFP to visualize the shape of the cell and stained with Yaksa. As shown in Fig. 4I-L and Fig. S3, Yaksa Ag localized at sites of cell-cell and cell-myotube contact. We did not detect
Right), and anti mg Ab and Yaksa (Lower Right). C Yaksa-positive cells form multinucleated cells shortly after replating. Thirty-six hours after induction, C2 cells sorted with Yaksa were replated, cultured for $6 \mathrm{~h}$, and then stained with anti-alpha actinin (green) and DAPI (red). Yaksa-positive cells form binuclear or multinucleated cells shortly after replating

this signal when using the isotype-matched control $\operatorname{IgG} 2 \mathrm{a}$ (Fig. S3).

\section{Discussion}

We established a novel monoclonal antibody, Yaksa that specifically recognizes prefusion myocytes. Yaksa provides a novel tool to clarify the molecular mechanisms of muscle cell fusion, because this antibody can mark or isolate prefusion myocytes among heterogeneously differentiating myoblasts.

So far, several surface markers for differentiating myoblasts have been reported including $\mathrm{N}-\mathrm{CAM}$ and M-cadherin (Blanco-Bose et al. 2001; Capkovic et al. 2008; Charrasse et al. 2007). However, either M-cadherin or NCAM, for example, is expressed on entire population of $\mathrm{C} 2$ cells after induction and neither marks a subpopulation of fusogenic C2 cells (data not shown). To our knowledge, a monoclonal antibody with which prefusion myocytes in mammal are sorted out alive, has not been reported yet although antiserum named as anti-M-24 was reported to react with prefusion myocytes in chick embryos 30 years ago (Friedlander and Fischman 1977). 
Fig. 4 Yaksa antigen was expressed in vivo.

A-C Transverse section of mouse embryo (E13.5) triplestained with Yaksa (A), antiDesmin (B) and DAPI (C). A dorsal quarter of embryo was shown. Arrow heads Desmin positive developing muscle. Signal in the peripheral of section is non-specific staining. D-F Transverse section of regenerating TA muscle 4 days after CTX injection was stained with Yaksa (green, E) and DAPI (red, F). G FACScan analysis of single cells prepared from regenerating TA muscle 3 days after CTX injection. Cells were stained with Yaksa (solid) or IgG2a (line).

H FACScan analysis of primary myoblasts prepared from gastrocnemius muscle. Cells were stained with Yaksa (solid) or IgG2a (line). I-L Yaksa antigen is expressed at site of cell-cell contact. Cultures of pMB expressing GFP (green, $\mathbf{K})$ were fixed and stained with biotinylated Yaksa \& StAvALEXA594 (red, J) and Hoechst (blue, L). Arrowheads Accumulation of Yaksa antigen at sites of cell-cell contact
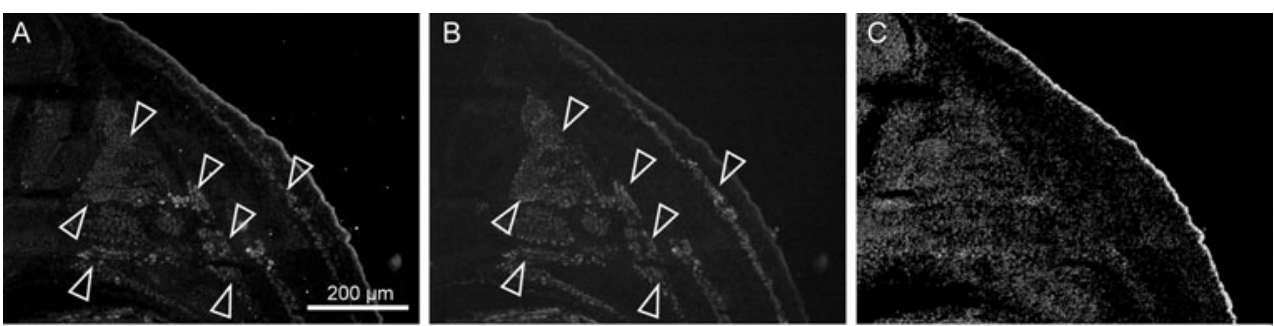

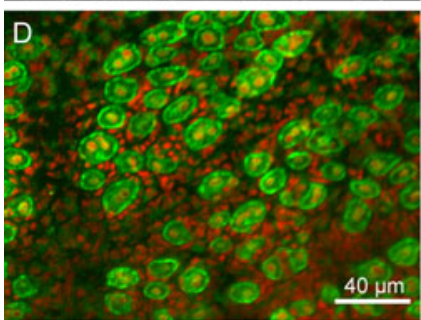

G

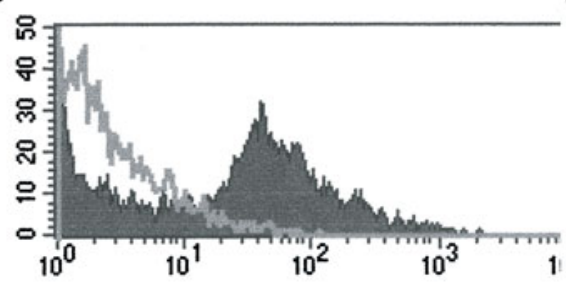

H
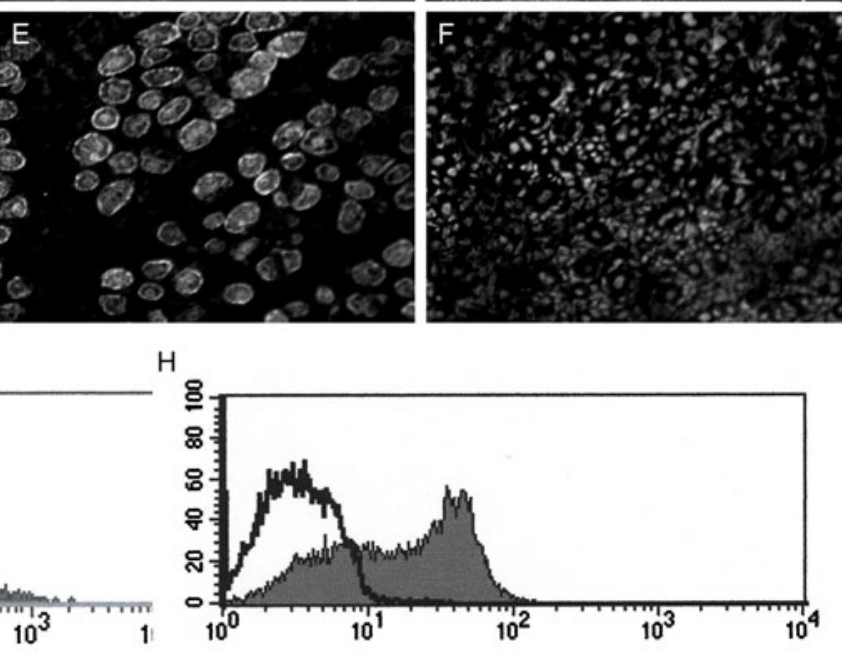
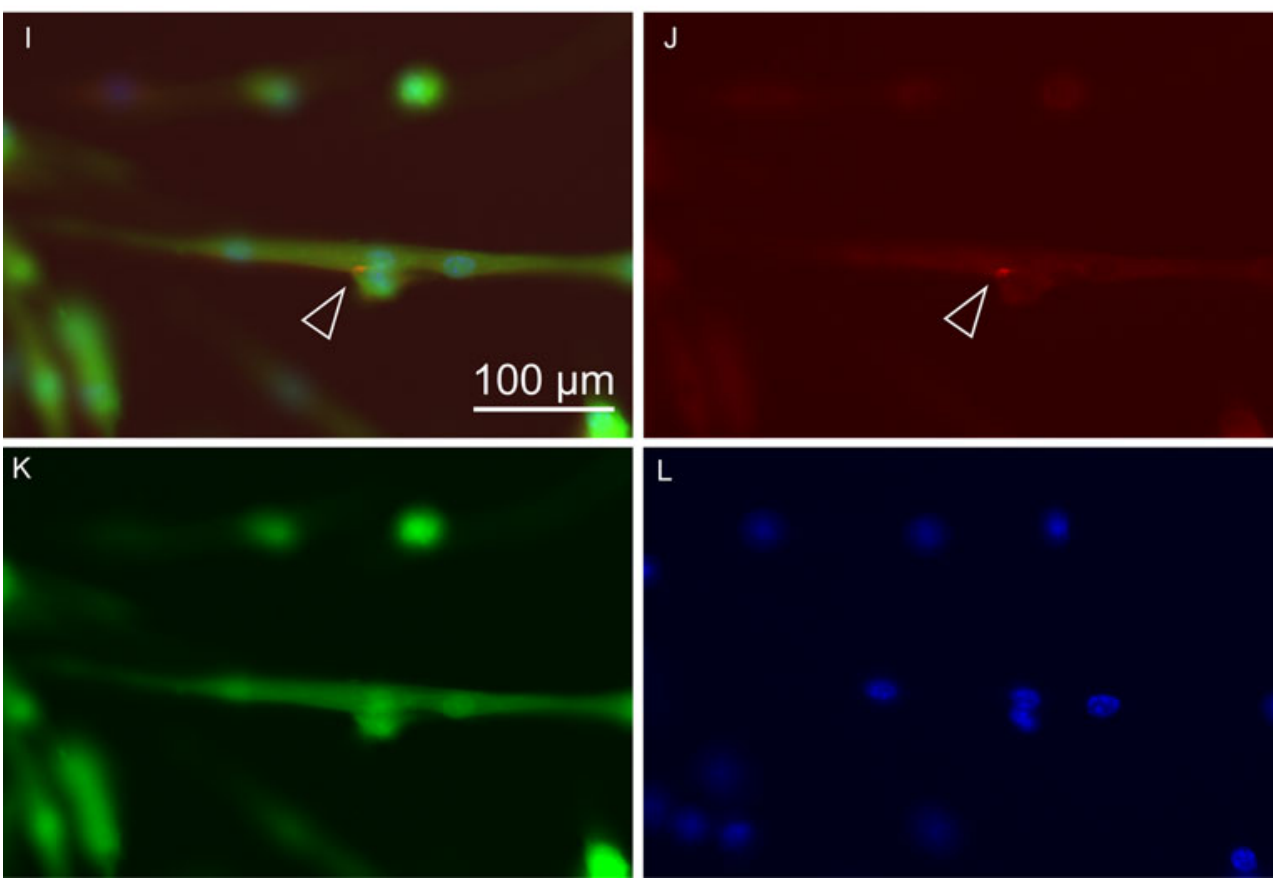

The results of our replating assay have two important implications for the fusion competence of cultured prefusion myocytes. First, most of Yaksa-positive cells fused with each other shortly after replating while Yaksa-negative cells scarcely generated multinucleated myotubes, suggesting that prefusion myocytes fuse among each other or with multinucleated myotubes. Second, C2 cells generate prefusion myocytes much earlier before myotube formation. In this paper, most replating assays were performed at $36 \mathrm{~h}$ after induction. However, Yaksa positivecells already existed as a small population (2-5\%) $24 \mathrm{~h}$ after induction, and they fused with each other within 6-8 h after replating (data not shown). This suggests that prefusion myocytes in cultured $\mathrm{C} 2$ cells could not contact each other efficiently resulting in failure of fusion, despite their fusion competency. 
Identification of Yaksa Ag is underway. Although Yaksa $\mathrm{Ag}$ is not identified yet, specific expression of Yaksa Ag on prefusion myocytes and localization at sites of cell-cell contact imply that Yaksa antigen plays positive roles in muscle differentiation and membrane fusion. In Drosophila, it was reported that a multiprotein complex, which was named FuRMAS (Fusion-Restricted Myogenic-Adhesive Structure), is assembled at the cell-cell contact site during fusion (Onel and Renkawitz-Pohl 2009; Kesper et al. 2007). FuRMAS consists of a ring structure of cell adhesion molecules, signaling proteins, and F-actin, and it has been proposed that FuRMAS serves as a signaling center, restricts the area of membrane fusion, and possibly triggers membrane breakdown, which leads to cellular fusion. Moreover, rafts, cholesterol-rich microdomains, likely play specific roles in cell fusion (Mukai et al. 2009). Although FuRMAS in mammalian myoblasts has not been reported yet, Yaksa would be a useful tool to clarify the molecular mechanisms of muscle cell fusion including identification of FuRMAS-related complexes in fusing cells. Identification of or molecules complexed with Yaksa Ag could be an alternative way to tackle the mechanisms of muscle cell fusion.

Acknowledgments This work was supported in part by a Grant-inAid for Scientific Research on Priority Areas and the project for realization of regenerative medicine from the Ministry of Education, Culture, Sports, Science and Technology, and in part by a research grant for Nervous and Mental Disorders from the Ministry of Health, Labour and Welfare.

Open Access This article is distributed under the terms of the Creative Commons Attribution Noncommercial License which permits any noncommercial use, distribution, and reproduction in any medium, provided the original author(s) and source are credited.

\section{References}

Andres V, Walsh K (1996) Myogenin expression, cell cycle withdrawal, and phenotypic differentiation are temporally separable events that precede cell fusion upon myogenesis. J Cell Biol 132:657-666

Blanco-Bose WE, Yao CC, Kramer RH, Blau HM (2001) Purification of mouse primary myoblasts based on alpha 7 integrin expression. Exp Cell Res 265:212-220

Buckingham M (2001) Skeletal muscle formation in vertebrates. Curr Opin Genet Dev 11:440-448

Buckingham M (2006) Myogenic progenitor cells and skeletal myogenesis in vertebrates. Curr Opin Genet Dev 16:525-532

Capkovic KL, Stevenson S, Johnson MC, Thelen JJ, Cornelison DD (2008) Neural cell adhesion molecule (NCAM) marks adult myogenic cells committed to differentiation. Exp Cell Res 314:1553-1565

Cerletti M, Shadrach JL, Jurga S, Sherwood R, Wagers AJ (2008) Regulation and function of skeletal muscle stem cells. Cold Spring Harb Symp Quant Biol 73:317-322
Charrasse S, Comunale F, Fortier M, Portales-Casamar E, Debant A, Gauthier-Rouviere C (2007) M-cadherin activates Rac1 GTPase through the Rho-GEF trio during myoblast fusion. Mol Biol Cell 18:1734-1743

Dzionek A, Fuchs A, Schmidt P, Cremer S, Zysk M, Miltenyi S, Buck DW, Schmitz J (2000) BDCA-2, BDCA-3, and BDCA-4: three markers for distinct subsets of dendritic cells in human peripheral blood. J Immunol 165:6037-6046

Friedlander M, Fischman DA (1977) Surface antigens of the embryonic chick myoblast: expression on freshly trypsinized cells. J Supramol Struct 7:323-338

Fujisawa-Sehara A, Hanaoka K, Hayasaka M, Hiromasa-Yagami T, Nabeshima Y (1993) Upstream region of the myogenin gene confers transcriptional activation in muscle cell lineages during mouse embryogenesis. Biochem Biophys Re Commun 191: $351-356$

Halevy O, Novitch BG, Spicer DB, Skapek SX, Rhee J, Hannon GJ, Beach D, Lassar AB (1995) Correlation of terminal cell cycle arrest of skeletal muscle with induction of p21 by MyoD. Science 267:1018-1021

Haralalka S, Abmayr SM (2010) Myoblast fusion in Drosophila. Exp Cell Res 316:3007-3013

Harlow E, Lane D (1988) Antibodies: a laboratory manual. Cold Spring Harbor, New York

Hashimoto N, Murase T, Kondo S, Okuda A, Inagawa-Ogashiwa M (2004) Muscle reconstitution by muscle satellite cell descendants with stem cell-like properties. Development 131:5481-5490

Hirata A, Masuda S, Tamura T, Kai K, Ojima K, Fukase A, Motoyoshi K, Kamakura K, Miyagoe-Suzuki Y, Takeda S (2003) Expression profiling of cytokines and related genes in regenerating skeletal muscle after cardiotoxin injection: a role for osteopontin. Am J Pathol 163:203-215

Horsley V, Pavlath GK (2004) Forming a multinucleated cell: molecules that regulate myoblast fusion. Cells Tissues Organs 176:67-78

Jansen KM, Pavlath GK (2008) Molecular control of mammalian myoblast fusion. Methods Mol Biol 475:115-133

Kesper DA, Stute C, Buttgereit D, Kreiskother N, Vishnu S, Fischbach KF, Renkawitz-Pohl R (2007) Myoblast fusion in Drosophila melanogaster is mediated through a fusion-restricted myogenic-adhesive structure (FuRMAS). Dev Dyn 236:404-415

Krauss RS (2010) Regulation of promyogenic signal transduction by cell-cell contact and adhesion. Exp Cell Res 316:3042-3049

Mermelstein CS, Portilho DM, Medeiros RB, Matos AR, EinickerLamas M, Tortelote GG, Vieyra A, Costa ML (2005) Cholesterol depletion by methyl- $\beta$-cyclodextrin enhances myoblast fusion and induces the formation of myotubes with disorganized nuclei. Cell Tissue Res 319:289-297

Miller JB (1990) Myogenic programs of mouse muscle cell lines: expression of myosin heavy chain isoforms, MyoD1, and myogenin. J Cell Biol 111:1149-1159

Moore CA, Parkin CA, Bidet Y, Ingham PW (2007) A role for the Myoblast city homologues Dock1 and Dock5 and the adaptor proteins Crk and Crk-like in zebrafish myoblast fusion. Development 134:3145-3153

Morgan JE, Partridge TA (2003) Muscle satellite cells. Int J Biochem Cell Biol 35:1151-1156

Mukai A, Kurisaki T, Sato SB, Kobayashi T, Kondoh G, Hashimoto N (2009) Dynamic clustering and dispersion of lipid rafts contribute to fusion competence of myogenic cells. Exp Cell Res 315:3052-3063

Nabeshima Y, Hanaoka K, Hayasaka M, Esumi E, Li S, Nonaka I (1993) Myogenin gene disruption results in perinatal lethality because of severe muscle defect. Nature 364:532-535

Ojima K, Uezumi A, Miyoshi H, Masuda S, Morita Y, Fukase A, Hattori A, Nakauchi H, Miyagoe-Suzuki Y, Takeda S (2004) 
Mac-1(low) early myeloid cells in the bone marrow-derived SP fraction migrate into injured skeletal muscle and participate in muscle regeneration. Biochem Biophys Re Commun 321: 1050-1061

Onel SF, Renkawitz-Pohl R (2009) FuRMAS: triggering myoblast fusion in Drosophila. Dev Dyn 238:1513-1525

Parker SB, Eichele G, Zhang P, Rawls A, Sands AT, Bradley A, Olson EN, Harper JW, Elledge SJ (1995) p53-Independent expression of p21Cip1 in muscle and other terminally differentiating cells. Science 267:1024-1027

Rochlin K, Yu S, Roy S, Baylies MK, Richardson BE (2010) Myoblast fusion: when it takes more to make one. Dev Biol 341:66-83

Rudnicki MA, Le Grand F, McKinnell I, Kuang S (2008) The molecular regulation of muscle stem cell function. Old Spring Harb Symp Quant Biol 73:323-331
Wada MR, Inagawa-Ogashiwa M, Shimizu S, Yasumoto S, Hashimoto N (2002) Generation of different fates from multipotent muscle stem cells. Development 129:2987-2995

Yaffe D, Saxel O (1977) Serial passaging and differentiation of myogenic cells isolated from dystrophic mouse muscle. Nature 270:725-727

Yoshida S, Fujisawa-Sehara A, Taki T, Arai K, Nabeshima Y (1996) Lysophosphatidic acid and bFGF control different modes in proliferating myoblasts. J Cell Biol 132:181-193

Yoshida N, Yoshida S, Koishi K, Masuda K, Nabeshima Y (1998) Cell heterogeneity upon myogenic differentiation: down-regulation of MyoD and Myf-5 generates 'reserve cells'. J Cell Sci 111:769-779 